TO BF PRESENTED AT THE 1995 PARTICLE ACCELERATOR CONFERENCE AND INTEFNATIONAL CONFERENCE ON HIGH ENERGY ACCELERATORS DALLAS, TEXAS MAY I-9, 1995

\title{
FIELD QUALITY CONTROL THROUGH THE PRODUCTION PHASE OF RHIC ARC DIPOLES*
}

BNL-61226

\author{
R. Gupta, A. Jain, S. Kahn, G. Morgan, P. Thompson, P. Wanderer, E. Willen \\ Brookhaven National Laboratory, Upton, NY 11973, USA
}

The field quality in the arc dipoles built thus far for the Relativistic Heavy Ion Collider (RHIC) not only meets machine requirements ${ }^{1}$ but is significantly better than that expected from scaling laws based on previous large scale superconducting magnet production for particle accelerators. In this paper we describe the evolution of the present cross section and the design philosophy that has led to these improvements. The techniques described here have been found quite efficient to adopt in the production environment, where schedule and cost considerations become important. Moreover, the techniques developed during the $R \& D$ program have resulted in making the saturation induced harmonics negligible, despite the fact that the iron is very close to the coil.

\section{INTRODUCTION}

The Relativistic Heavy Ion Collider being built at the Brookhaven National Laboratory will require 288 superconducting dipole magnets in the arcs. The cross section of these magnets is shown in Fig. 1 and the basic design parameters are given in Table 1. About half of them are already built by Northrop-Grumman Corporation and the field harmonics are measured ${ }^{2}$. The field harmonics are defined in the following relation:

$$
B_{y}+i B_{x}=10^{-4} B_{0} \sum_{n=0}^{\infty}\left[b_{n}+i a_{n}\right]\left(\frac{x+i y}{R_{0}}\right)^{n},
$$

where $B_{x}$ and $B_{y}$ are the components of the field at $(x, y)$ and $B_{0}$ is the central field. $a_{n}$ are the skew harmonics and $b_{n}$ are the normal. $R_{0}$ is the normalization radius which is chosen to be $25 \mathrm{~mm}$ in these magnets.

Table 1: Basic design parameters of $R H I C$ arc dipoles

\begin{tabular}{|c|c|}
\hline Coil inner, outer radius & $40 \mathrm{~mm}, 50 \mathrm{~mm}$ \\
\hline Yoke inner, outer radius & $59.7 \mathrm{~mm}, 133.4 \mathrm{~mm}$ \\
\hline Field, current at injection & $0.40 \mathrm{~T}, 0.57 \mathrm{kA}$ \\
\hline Maximum design field, current & $3.46 \mathrm{~T}, 5.09 \mathrm{kA}$ \\
\hline Computed quench at $4.5^{\circ} \mathrm{K}$ & $8.25 \mathrm{kA}$ \\
\hline Magnetic length at 3.46 Tesla & $9.44 \mathrm{~m}$ \\
\hline
\end{tabular}

The major sources of harmonic content in superconducting magnets are : (a) Geometric multipoles due to a non-ideal magnet geometry, (b)Persistent current induced multipoles due to the superconducting properties of the cable, (c) Saturation induced multipoles due to non-linear properties of the iron yoke, (d) Coil deformation multipoles due to changes in the shape of the collared coil due to Lorentz forces.

LISTRIBUTION OF THIS DOCUMENT IS

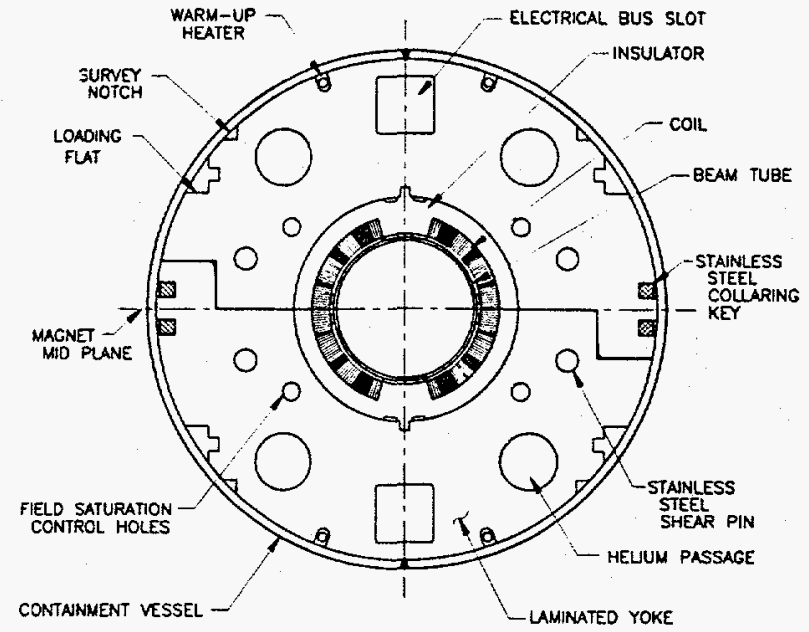

Figure 1: The cross section of the RHIC arc dipoles.

\section{COIL DESIGN ITERATIONS}

To obtain a high field quality such as that measured in RHIC magnets, the conductors must be placed at the appropriate location to an accuracy of $\sim 50 \mu \mathrm{m}\left(0.002^{\prime \prime}\right)$. However, after the coils are manufactured, they go through significant deformation during curing, collaring and cool down processes and there is no direct control on where an individual turn will exactly go. The mechanical deformation in the coil and iron shape during manufacturing is not calculable to a combined accuracy of $50 \mu \mathrm{m}$. To overcome this limitation, we empirically remove the influence of these distortions by calculating the offsets in measured field harmonics from the computed values after including all known sources. These offsets are subtracted out during the cross section iterations. In addition, the required changes in the iterated design are specified in terms of relative changes in dimensions as compared to the previous design so that the errors in tooling etc. get subtracted out.

The successful outcome of this approach is clear from Table 2 where the measured averages for the allowed harmonics are given for the Prototype, Phase 1, Phase $1 \mathrm{~A}$ and Phase 2 dipoles. The Phase 1 cross section was the initial cross section used in the first 19 industrybuilt magnets which was based on the cross section used in the last two prototype magnets built at BNL. The coil midplane gap was deliberately made larger by 0.05 $\mathrm{mm}$ than the required ${ }^{3}$ minimum value of $0.10 \mathrm{~mm}$. This was to compensate for a potential change in field quality associated with the change in tooling between the prototype and industry built magnets. An adjustment in the midplane gap is much more powerful than an alternate method of adjusting coil pole shim, particularly 
for the crucial $b_{4}$ harmonic. In fact, this adjustment got quickly implemented in the Phase $1 \mathrm{~A}$ cross section (used in the next 86 magnets), when the coil midplane gap was changed from $0.15 \mathrm{~mm}$ to $0.10 \mathrm{~mm}$ to reduce $b_{4}$. The change in midplane gap back to $0.15 \mathrm{~mm}$ together with a change in one wedge by $63.5 \mu \mathrm{m}$ was incorporated in the Phase 2 cross section. The current production is proceeding on this design and 24 dipoles are included in Table 2. In all cases good agreement has been found between the calculations and measurements.

Table 2: The average and RMS values of field harmonics in various cross section designs for $R H I C$ arc dipoles. The b2 harmonic is given at the maximum field (3.46 T) and the other harmonics at injection (0.4 Tesla). The measured warm cold correlation of 40 magnets is used to estimate harmonics in 129 magnets measured warm.

\begin{tabular}{|c|c|c|c|c|}
\hline Design & $b_{2}$ & $b_{4}$ & $b_{6}$ & $b_{8}$ \\
\hline Prototype & $1.3 \pm 0.8$ & $0.3 \pm 0.2$ & $-0.1 \pm 0.05$ & $0.40 \pm 0.03$ \\
\hline Phase 1 & $0.4 \pm 1.6$ & $-1.0 \pm 0.4$ & $-0.38 \pm 0.09$ & $0.20 \pm 0.06$ \\
\hline Phase 1A & $1.2 \pm 1.2$ & $-0.4 \pm 0.30$ & $-0.10 \pm 0.08$ & $0.24 \pm 0.03$ \\
\hline Phase 2 & $-0.3 \pm 1.3$ & $0.1 \pm 0.32$ & $-.21 \pm 0.09$ & $0.00 \pm 0.03$ \\
\hline
\end{tabular}

Table 3: The computed changes in the values of harmonics produced by a systematic azimuthal error of $+25 \mu \mathrm{m}\left(0.001^{\prime \prime}\right)$ in crucial parts in RHIC arc dipales.

\begin{tabular}{|c|c|c|c|c|}
\hline Parameter & $\delta b_{2}$ & $\delta b_{4}$ & $\delta b_{6}$ & $\delta b_{8}$ \\
\hline Wedge 1 & -0.98 & -0.122 & 0.061 & 0.043 \\
\hline Wedge 2 & 0.69 & 0.423 & 0.022 & -0.050 \\
\hline Wedge 3 & 1.42 & -0.090 & -0.068 & 0.041 \\
\hline Pole Width & -1.11 & 0.154 & -0.039 & 0.014 \\
\hline Midplane Gap & -1.68 & -0.557 & -0.156 & -0.050 \\
\hline
\end{tabular}

In RHIC magnets, the specified tolerances in the dimensions of the most crucial parts are typically $\pm 25 \mu \mathrm{m}$ $\left(0.001^{\prime \prime}\right)$. In Table 3 we list the harmonics produced by a systematic $+25 \mu m$ azimuthal error in the three wedges, pole width and the coil midplane gap. The coil midplane can not have such a large error and is given for $25 \mu \mathrm{m}$ only for consistency. Moreover, field harmonics are also created by other parts used in the magnets, such as the errors in yoke dimensions etc., but they are generally expected to have a smaller impact on harmonics. In a few magnets the spacers used between the coil and iron were just outside the thickness tolerance and caused a noticeable change in the transfer function ${ }^{2}$.

The harmonics in Table 2 are comparable to those in Table 3. This suggests that the harmonies are not limited by the design and that these are probably the best harmonics one may hope for with reasonable values of mechanical tolerances and normal variations in the manufacturing process.

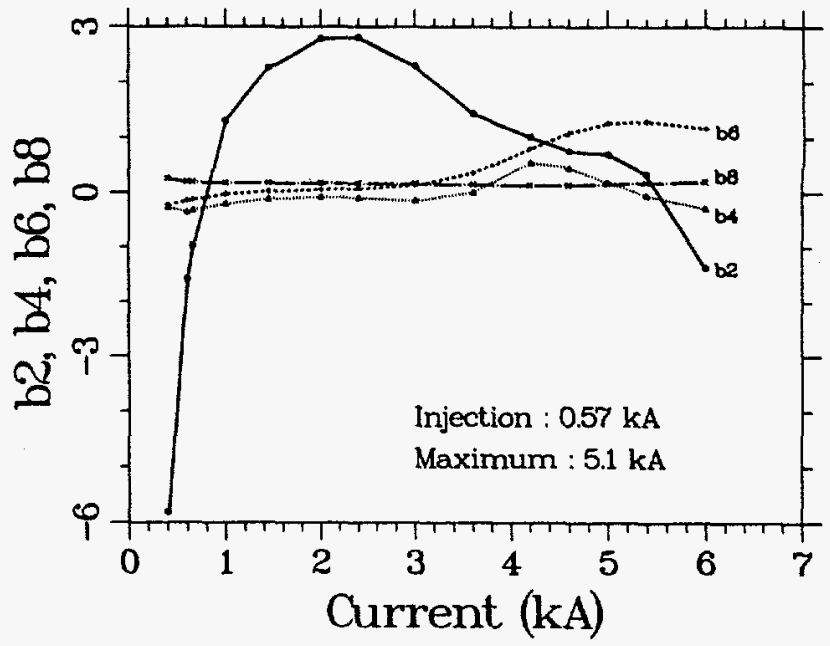

Figure 2: The measured current dependence of harmonics during up ramp (and 20 second wait) in $R H I C$ arc dipoles.

\section{YOKE DESIGN ITERATIONS}

In RHIC magnet designs, the yoke has been brought very close to the coil in an attempt to get the maximum contribution from the iron. The conventional wisdom against such attempts in the past had been that the saturation induced harmonics would become unavoidably large. However, the results from RHIC magnets show that despite a high $(\sim 35 \%)$ contribution from iron $(50 \%$ to $100 \%$ higher than those used in major accelerator magnets) the saturation induced harmonics can be controlled to a small value. In Fig. 2, we have plotted the average values of field harmonics as a function of current during the up ramp in 129 magnets. Only 40 of them are actually measured cold and for others an already well established warm-cold correlation from 40 magnets is used ${ }^{2}$. The dominant source for the current dependence below $2 \mathrm{kA}$ is the persistent current in the superconductors, whereas above $2 \mathrm{kA}$ it is the iron saturation and the change in coil and iron shape when Lorentz forces are unloading the pre-compression on the coil. In the present cross section, the deviation from the average in the design range of operation is \pm 2.5 unit in $b_{2}$ and \pm 0.4 unit in $b_{4}$. These values are comparable to those generated by $\pm 25 \mu m$ error in more than one part (Table 3). Except for the $b_{2}$ harmonic, all harmonics were optimized at the injection field. The $b_{2}$ harmonic was minimized at the maximum field where it is 2.5 units higher than at the injection. In addition, the variation in them is also minimized at the intermediate fields. The saturation induced $b_{6}$ harmonic was not optimized, as long as it did not become too large.

In Fig. 3, we show the current dependence in the $b_{2}$ and $b_{4}$ harmonics in various yoke designs. The harmonics are an average of up and down ramps to remove the persistent current induced harmonics to first order. Also, an offset is added in each magnet so that they coincide at $2 \mathrm{kA}$ for easy comparison. One can see an order of magnitude improvement. 


\section{DISCLAIMER}

This report was prepared as an account of work sponsored by an agency of the United States Government. Neither the United States Government nor any agency thereof, nor any of their employees, make any warranty, express or implied, or assumes any legal liability or responsibility for the accuracy, completeness, or usefulness of any information, apparatus, product, or process disclosed, or represents that its use would not infringe privately owned rights. Reference herein to any specific commercial product, process, or service by trade name, trademark, manufacturer, or otherwise does not necessarily constitute or imply its endorsement, recommendation, or favoring by the United States Government or any agency thereof. The views and opinions of authors expressed herein do not necessarily state or reflect those of the United States Government or any agency thereof. 


\section{DISCLAIMER}

Portions of this document may be illegible in electronic image products. Images are produced from the best available original document. 

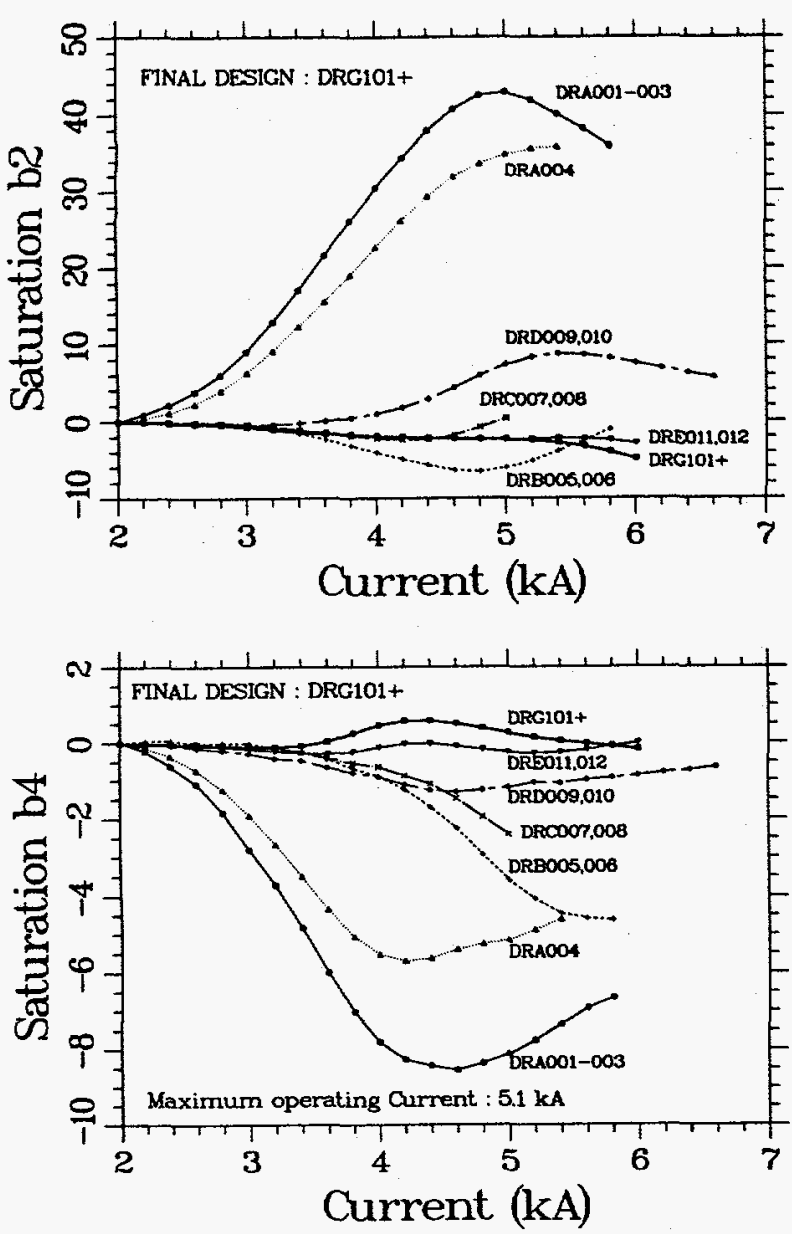

Figure 3: The current dependence of $b_{2}$ and $b_{4}$ in various designs of RHIC arc dipoles after removing the persistent current effects.

The first yoke design was used in four (DRA series) magnets - the last one (DRA004) had a minor modification at the yoke outer surface. This cross section had a coil locating notch at the pole and a small coil-to-yoke gap of $5 \mathrm{~mm}$. Both of these features contributed to a large saturation. In the second design, used in two (DRB series) magnets, the coil-to-yoke gap was increased to 10 $\mathrm{mm}$ and the notch was moved to the midplane to reduce saturation. Then in the next two (DRC series) magnets, the material of the yoke-yoke alignment key was changed from non-magnetic stainless steel to magnetic steel. This made $b_{2}$ very small and $b_{4}$ significantly smaller but still large enough to require the external decapole correctors. Moreover, in order to improve the coil pole definition/location, it was decided to move the coil locating notch back to the pole from the midplane. At this stage, the yoke cross section was completely redesigned for the next two (DRD series) magnets and $b_{4}$ saturation was made small enough to consider dropping the decapole correctors from the lattice. However the pole notch, as expected, gave a relatively large $b_{2}$ saturation. Finally, a saturation control hole was added at a critical location near the yoke inner radius in the last two prototype magnets (DRE series) to make saturation induced $b_{2}$ and $b_{4}$ practically zero. However, in the DRG series yoke design, which is used in the industrially built magnets, the material of the yoke-yoke alignment key was changed from the magnetic low carbon steel to non-magnetic stainless steel to match the thermal contraction of the shell during cool down. The location of the saturation control hole was changed to compensate for extra saturation introduced by this change.

In the RHIC arc dipoles, the cold mass is not vertically centered in the cryostat. At high field this creates a skew quadrupole harmonic of $\sim 2$ units. In the present design, the yoke weight difference between the top and bottom halves is adjusted to compensate for this effect.

\section{CONCLUSIONS}

The R\&D program carried out at Brookhaven has resulted in a significant improvement in field quality of the critical arc dipole magnets for RHIC. In Fig. 4 the net field error on the midplane in these magnets is compared with the similar aperture dipoles for other large accelerators.

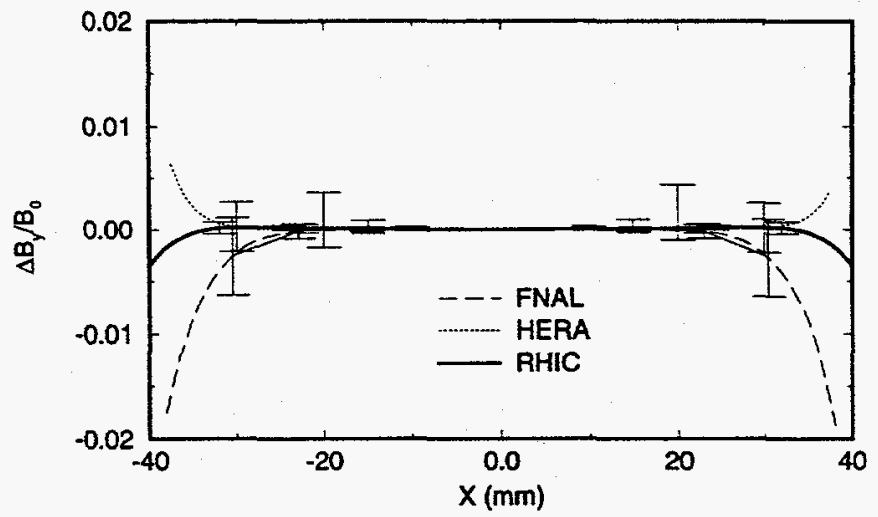

Figure 4: The field error at top operating field on the midplane of the $80 \mathrm{~mm}$ aperture $R H I C$ arc dipoles compared with $76 \mathrm{~mm}$ aperture HERA and $76.2 \mathrm{~mm}$ aperture Tevatron dipoles. The error bars show the RMS variations. (Courtesy S. Peggs and J. Wei).

\section{REFERENCES}

1. J. Wei, et al., "Field Quality Evaluation of the Superconducting Magnets for the Relativistic Heavy Ion Collider", this conference.

2. P. Wanderer, et al., "Construction and Testing of Arc Dipoles and Quadrupoles for the Relativistic Heavy Ion Collider (RHIC) at $\mathrm{BNL}^{n}$, this conference.

3. R. Gupta, et al., "Field Quality Improvements in Superconducting Magnets for RHIC", 1994 European Particle Accelerator Conference.

4. S. Peggs, "Private Communication."

${ }^{*}$ Work supported by the U.S. Department of Energy under the contract No. DE-AC02-76CH00016. 